\title{
CMOS Ultra-Wideband Low Noise Amplifier Design
}

\author{
K. Yousef, ${ }^{1}$ H. Jia, ${ }^{2}$ R. Pokharel, ${ }^{3}$ A. Allam, ${ }^{1}$ M. Ragab, ${ }^{1}$ H. Kanaya, ${ }^{3}$ and K. Yoshida ${ }^{3}$ \\ ${ }^{1}$ Electronics and Communications Engineering Department, Egypt-Japan University of Science and Technology, \\ New Borg Al-Arab, 21934 Alexandria, Egypt \\ ${ }^{2}$ E-JUST Center, Kyushu University, Nishi-ku, Fukuoka 819-0395, Japan \\ ${ }^{3}$ Graduate School of ISSE, Kyushu University, Nishi-ku, Fukuoka 819-0395, Japan
}

Correspondence should be addressed to K. Yousef; khalil.yousef@ejust.edu.eg

Received 29 November 2012; Accepted 26 March 2013

Academic Editor: Mohammad S. Hashmi

Copyright $\odot 2013 \mathrm{~K}$. Yousef et al. This is an open access article distributed under the Creative Commons Attribution License, which permits unrestricted use, distribution, and reproduction in any medium, provided the original work is properly cited.

This paper presents the design of ultra-wideband low noise amplifier (UWB LNA). The proposed UWB LNA whose bandwidth extends from $2.5 \mathrm{GHz}$ to $16 \mathrm{GHz}$ is designed using a symmetric 3D RF integrated inductor. This UWB LNA has a gain of $11 \pm 1.0 \mathrm{~dB}$ and a NF less than $3.3 \mathrm{~dB}$. Good input and output impedance matching and good isolation are achieved over the operating frequency band. The proposed UWB LNA is driven from a $1.8 \mathrm{~V}$ supply. The UWB LNA is designed and simulated in standard TSMC $0.18 \mu \mathrm{m}$ CMOS technology process.

\section{Introduction}

CMOS technology is one of the most prevailing technologies used for the implementation of radio frequency integrated circuits (RFICs) due to its reduced cost and its compatibility with silicon-based system on chip [1]. The use of ultra-wideband (UWB) frequency range $(3.1-10.6 \mathrm{GHz})$ for commercial applications was approved in February 2002 by the Federal Communications Commission. Low cost, reduced power consumption, and transmission of data at high rates are the advantages of UWB technology. UWB technology has many applications such as wireless sensor and personal area networks, ground penetrating radars, and medical applications [2].

Low noise amplifier is considered the backbone of the UWB front-end RF receiver. It is responsible for signal reception and amplification over the UWB frequency range. LNA has many desired design specifications such as low and flat noise figure, high and flat power gain, good input and output wide impedance matching, high reverse isolation, and reduced DC power consumption $[1,3]$.

Nowadays one of the most suitable configurations suggested for LNA implementation is current reuse cascaded amplifier. This LNA configuration can attain low DC power consumption, high flattened gain, minimized NF, and excellent reverse isolation while achieving wide input and output impedance matching [1-3].

Radio frequency integrated inductors play a significant role in radio frequency integrated circuits (RFICs) implementation. Design, development, and performance improvement of RF integrated inductors represent a challenging work. Achieving high integration level and cost minimization of RFICs are obstructed because of the difficulties facing the RF integrated inductors designers which are related to obtaining high quality factors [4-6].

In this paper, the implementation of LNAs using 3D integrated inductors will be investigated. A symmetric 3D structure is proposed as a new structure of integrated inductors for RFICs.

This paper discusses the design procedure of current reuse cascaded UWB LNA and its bandwidth expansion. In addition, the employment of suggested symmetric 3D $\mathrm{RF}$ integrated inductor will be demonstrated. This paper is organized as follows. Section 2 introduces the suggested UWB LNA circuit. Section 3 gives simulation results and discussion. Conclusion is driven in Section 4 


\section{Circuit Description}

As shown in Figure 1, the proposed UWB LNA is a current reuse cascaded core based on a common source topology with a shunt resistive feedback technique implemented over the input stage.

This current reuse cascaded amplifier achieved good wideband input impedance matching through the use of source degeneration input matching technique. Figure 2 shows the small signal equivalent circuit of this LNA input stage. The input port of this UWB LNA is desired to match source impedance $R_{s}$ at resonance frequency $\omega_{o}$. This matching circuit bandwidth is defined through the quality factors of source degeneration and gain-peaking inductors $\left(L_{s}\right.$ and $\left.L_{g}\right)$ where the input impedance is given by

$$
\begin{aligned}
Z_{\text {in }} & =j \omega\left(L_{s}+L_{g}\right)+\frac{1}{j \omega C_{\mathrm{gs}}}+\omega_{T} L_{s} \\
& =j \omega\left(L_{s}+L_{g}\right)+\frac{1}{j \omega C_{\mathrm{gs}}}+R_{s},
\end{aligned}
$$

where $Z_{\text {in }}$ is the UWB LNA input impedance and $\omega_{T}$ is the current-gain cut-off frequency, where $\omega_{T}=g_{m} / C_{g s}$ and $g_{m}$ and $C_{g s}$ are the input stage transconductance and gate-source capacitance, respectively. $V_{s}$ represents the RF signal source. $R_{s}$ is the output impedance of $V_{s}$.

Although the shunt resistive feedback loop leads to LNA noise performance degradation [7], it is widely used in recently proposed LNAs due to its superior wideband characteristics. Shunt capacitive-resistive feedback technique is employed to widen the input-matching bandwidth and increase the LNA stability.

Shunt-peaked amplifiers are known to have wide gain bandwidth and high low frequency power gain [8]. To have a high flattened gain of the proposed UWB LNA, shuntpeaking technique is used. In addition the gate-peaking technique is used to enhance the LNA gain at high frequencies. Besides the shunt- and gate-peaking techniques, the shunt resistive feedback loop is used in gain flattening $[2,8]$. The LNA approximate gain is given by

$$
\begin{aligned}
A & \cong \frac{V_{\text {out }}}{V_{s}} \\
& \cong \frac{g_{m 1} g_{m 2}\left[R_{L} / /\left(R_{d 2}+S L_{d 2}\right)\right]\left[S L_{d 1}\right]}{2 \cdot S C_{g s 1}\left[S\left(L_{s 1}+L_{g 1}\right)+1 / S C_{\text {gs } 1}\right]} .
\end{aligned}
$$

Ultra-wideband applications require good noise performance in addition to high and flat gain. Low noise design techniques which are suitable for narrowband applications cannot be used for wideband applications. Main contribution of cascaded matched stages noise figure is due to first stage [9]. The reduction of noise figure of input stage will lead to the reduction of the overall noise figure of the proposed design. Optimization and control of factors affecting the NF will improve this UWB LNA noise performance. An equivalent circuit of the input stage for noise factor calculation is shown in Figure 3 [1].
An estimated value of the noise figure $\left(\mathrm{NF}=10 \log _{10} f\right)$ of this topology is given in [1] where $f$ is the noise factor of the UWB LNA. The noise factor $f$ can be given by

$$
\begin{aligned}
f= & +\frac{R_{g}+R_{\mathrm{lg}}+R_{\mathrm{ss}}+R_{\mathrm{ls}}}{R_{s}}+\frac{\delta \alpha \omega^{2} C_{\mathrm{gs} 1}^{2} R_{s}}{5 g_{m 1}} \\
& +\frac{R_{\mathrm{FB}}\left(\left(L_{g 1}+L_{s 1}\right) C_{\mathrm{gs} 1}\right)^{2}}{R_{s}\left(g_{m 1} R_{\mathrm{FB}}-1\right)^{2}} \\
& \cdot\left|s^{2}+s\left(\frac{\omega_{o, \mathrm{rfbn}}}{Q_{\mathrm{rfbn}}}\right)+\omega_{o, \mathrm{rfbn}}^{2}\right|^{2} \\
& +\frac{\gamma g_{m 1}\left(R_{\mathrm{FB}}+R_{s}\right)^{2}\left(\left(L_{g 1}+L_{s 1}\right) C_{\mathrm{gs} 1}\right)^{2}}{\alpha R_{s}\left(g_{m 1} R_{\mathrm{FB}}-1\right)^{2}} \\
& \cdot\left|s^{2}+s\left(\frac{\omega_{o, \mathrm{dn}}}{Q_{\mathrm{dn}}}\right)+\omega_{o, \mathrm{dn}}^{2}\right|^{2}, \\
f=1+ & \frac{R_{g}+R_{\mathrm{lg}}+R_{\mathrm{ss}}+R_{\mathrm{ls}}}{R_{s}}+f_{\mathrm{gn}}+f_{\mathrm{rfbn}}+f_{\mathrm{dn}},
\end{aligned}
$$

where

$$
\begin{gathered}
\omega_{o, \mathrm{rfbn}}=\sqrt{\frac{1+g_{m 1} R_{s}}{\left(L_{g 1}+L_{s 1}\right) C_{\mathrm{gs} 1}}} \\
Q_{\mathrm{rfbn}}=\frac{1}{R_{s}+\omega_{T 1} L_{s 1}} \cdot \sqrt{\frac{\left(1+g_{m 1} R_{s}\right)\left(L_{g 1}+L_{s 1}\right)}{C_{\mathrm{gs} 1}}}, \\
Q_{\mathrm{dn}}=\frac{\omega_{o, \mathrm{rfbn}}=\sqrt{\frac{1}{\left(L_{g 1}+L_{s 1}\right) C_{\mathrm{gs} 1}}},}{1}, \sqrt{\frac{\left(L_{g 1}+L_{s 1}\right)}{\left.C_{\mathrm{gB} 1}\right)+\omega_{T 1} L_{s 1}}},
\end{gathered}
$$

where $f_{\mathrm{gn}}, f_{\mathrm{dn}}$, and $f_{\mathrm{rfbn}}$ are gate, drain, and feedback resistor noise factors, respectively and $\alpha, \delta$, and $\gamma$ are constants equal to $0.85,4.1$, and 2.21 , respectively.

It is clear from (4) that, to reduce the noise figure, high quality factors of $L_{s 1}$ and $L_{g 1}$ are desired. It can also be noted that the noise factor is inversely proportional to feedback resistor $R_{f}$. In other words, weak feedback topology decreases the noise factor value while strong feedback implementation degrades the noise performance of the suggested UWB LNA.

In addition, the noise factor formula given by (4) states that the noise figure is also inversely proportional to the transconductance of the input stage $\left(g_{m 1}\right)$. This goes along with the known fact that noise performance trades off with power consumption.

For output matching, the series resonance of the shunt peaking technique is used to match the proposed UWB LNA to the load impedance $R_{L}$ while the series drain resistance $R_{d 2}$ is used to extend the output matching bandwidth.

This proposed UWB LNA (LNA1) has an operating bandwidth of 3.1-10.6 GHz. The proposed LNA2 whose schematic 


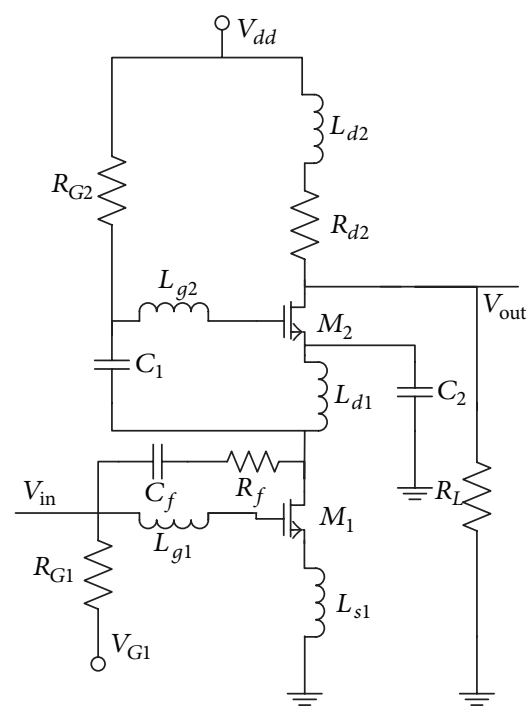

Figure 1: Current reuse UWB LNA (LNA1).

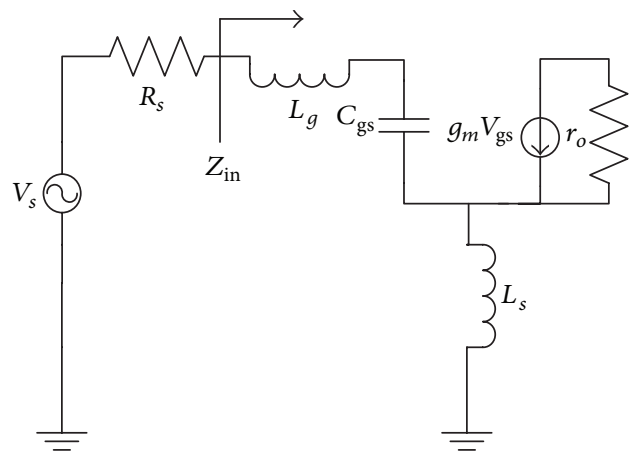

FIGURE 2: Input stage small signal equivalent circuit.

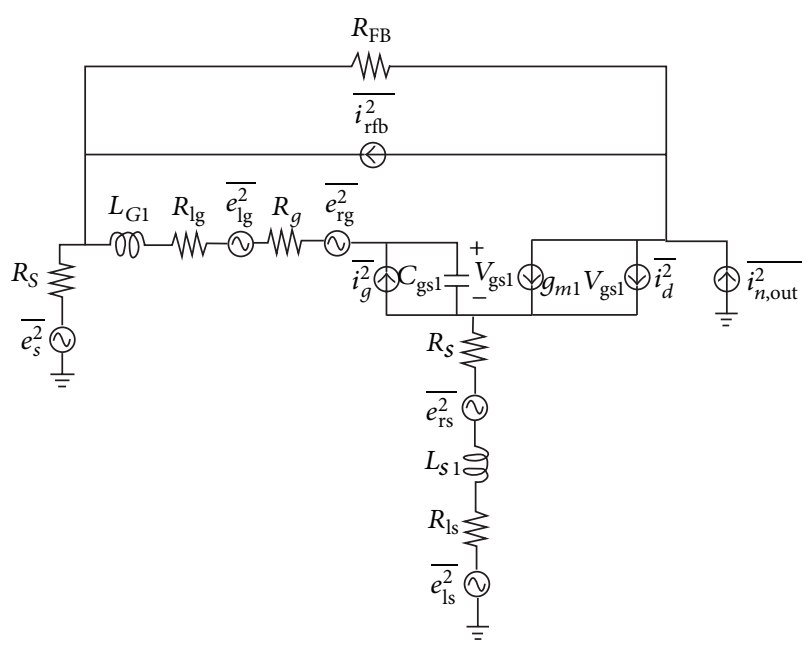

Figure 3: Equivalent circuit of the fisrt stage for noise calculation [1].

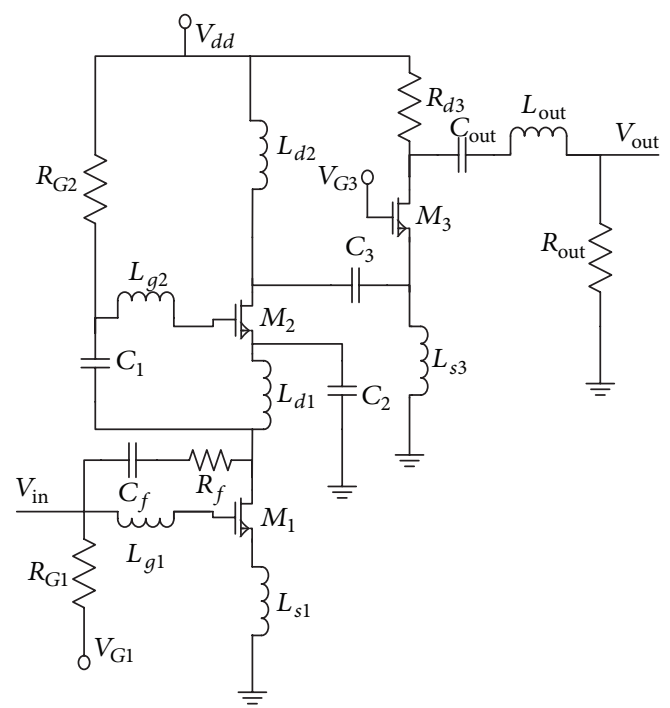

FIGURE 4: Schematic circuit of LNA2.

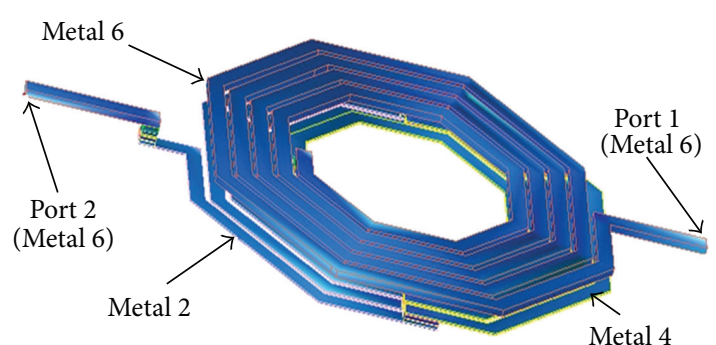

Figure 5: 3D view of the symmetric 3D proposed structure.

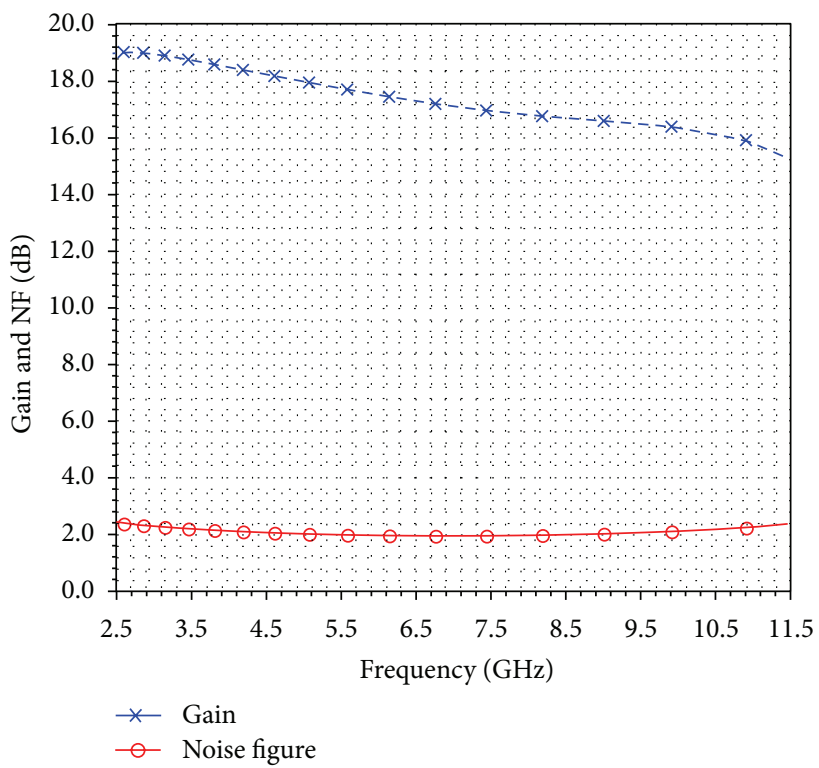

Figure 6: $S_{21}(\mathrm{~dB})$ and NF (dB) of LNA1. 


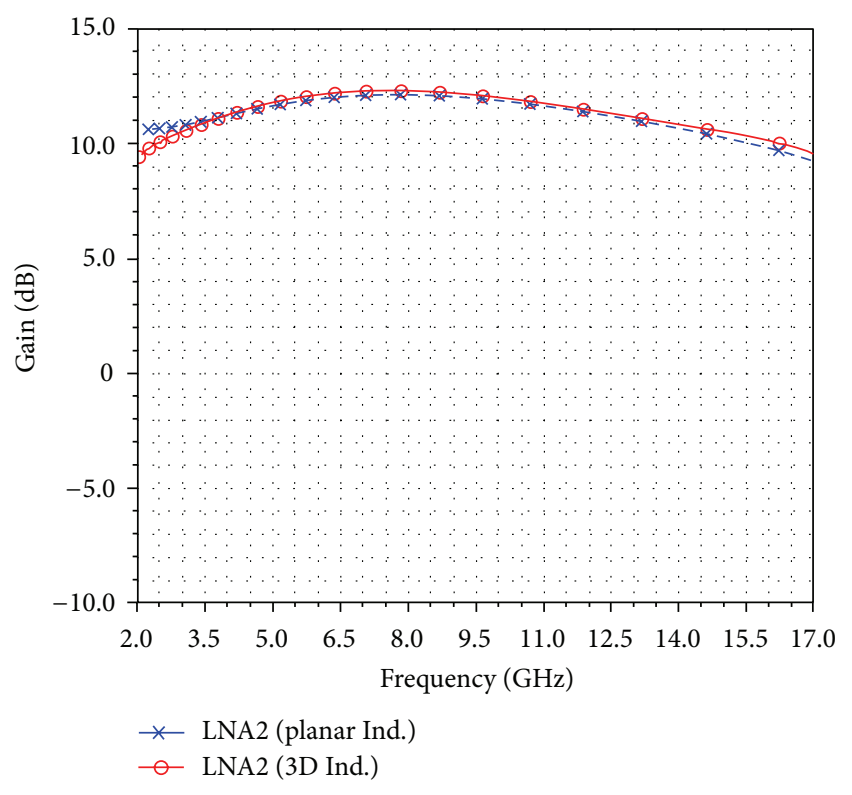

Figure 7: $S_{21}(\mathrm{~dB})$ of LNA2.

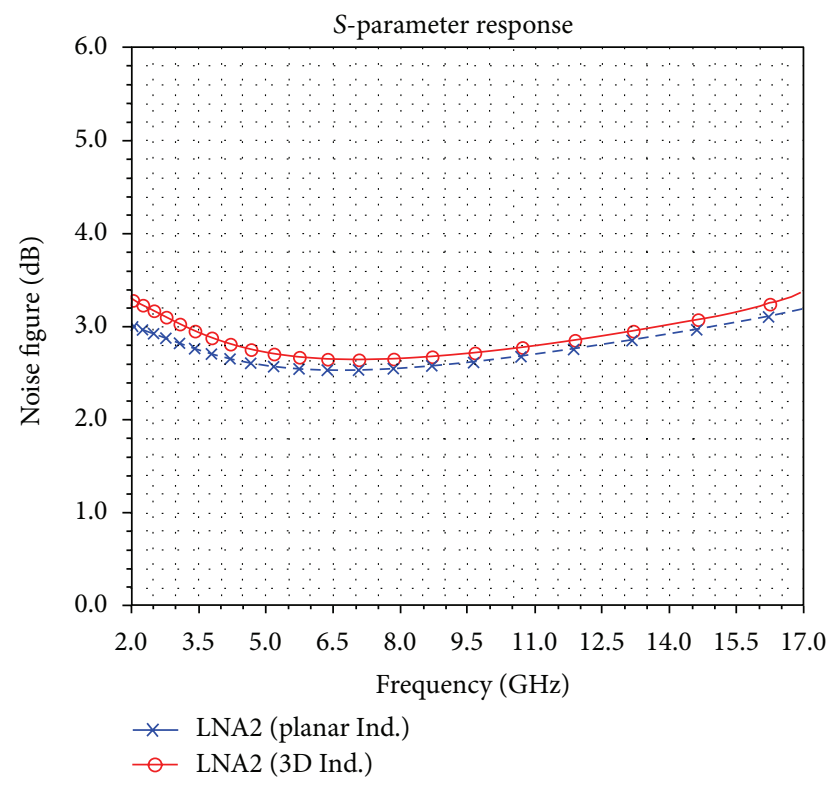

Figure 8: NF (dB) of LNA2.

circuit is shown in Figure 4 is an extended version of LNA1. It has a wider operating band of frequency which extends from $2.5 \mathrm{GHz}$ to $16 \mathrm{GHz}$.

Input impedance match has a special importance and consideration especially in wideband sensitive circuits design. Input impedance matching bandwidth is broadened by the use of a weaker shunt capacitive-resistive feedback loop which mainly leads to quality factor reduction of the input matching circuit. Weakness of shunt feedback strength not only reduces the input reflection coefficient over this wide bandwidth but it also reduces the input side injected thermal noise which decreases the proposed LNA2 noise

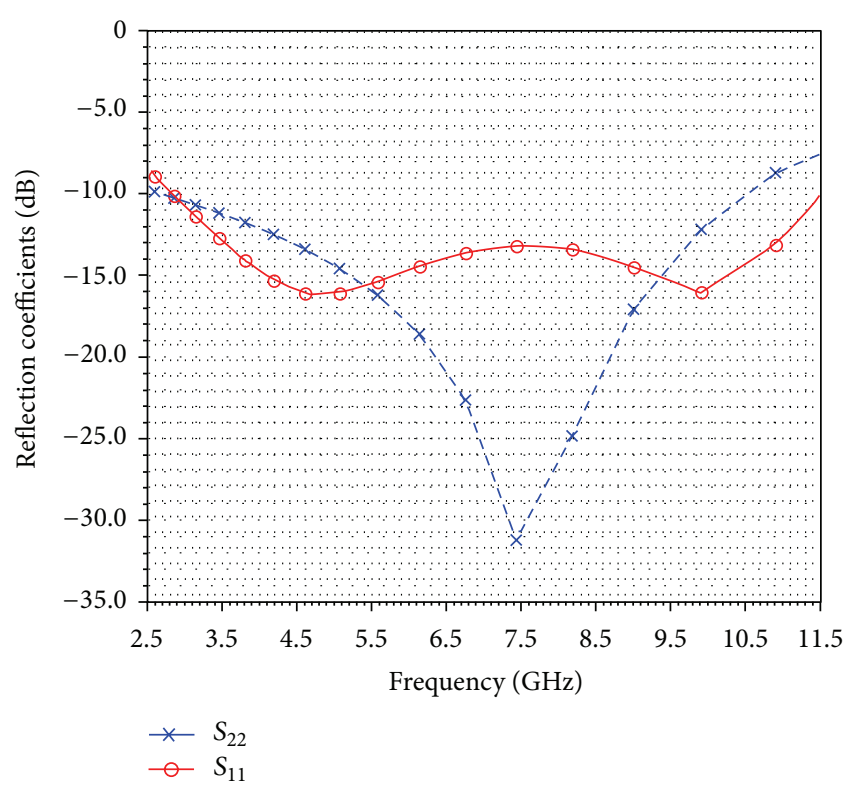

Figure 9: $S_{11}(\mathrm{~dB})$ and $S_{22}(\mathrm{~dB})$ of LNA1.

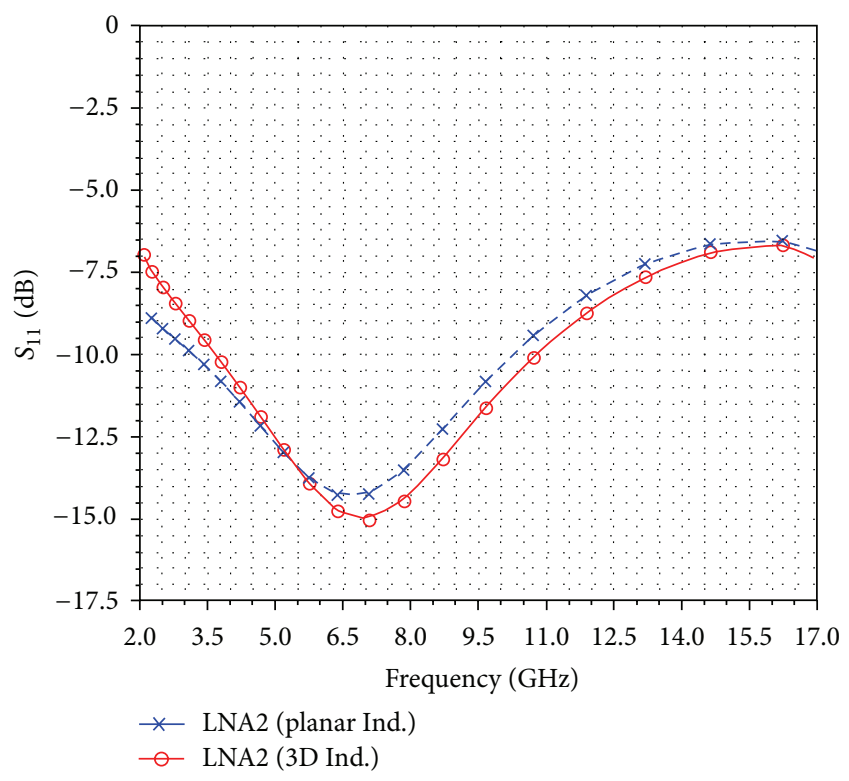

Figure 10: $S_{11}(\mathrm{~dB})$ of LNA2.

figure indicating the enhanced noise performance of the suggested design.

Shunt-peaking technique increases the low frequency gain and hence decreases the gain flatness while having a wide operating bandwidth. In spite of shunt-peaking drawbacks, it mainly facilitates LNA output impedance to load matching. LNA2 bandwidth extension and gain flatness over its operating band of frequency are achieved through the removal of shunt peaking. Moreover the control of gate peaking is used to enhance the current reuse amplifier core gain.

For wideband output impedance matching, a unity common gate (CG) matching topology in addition to series 


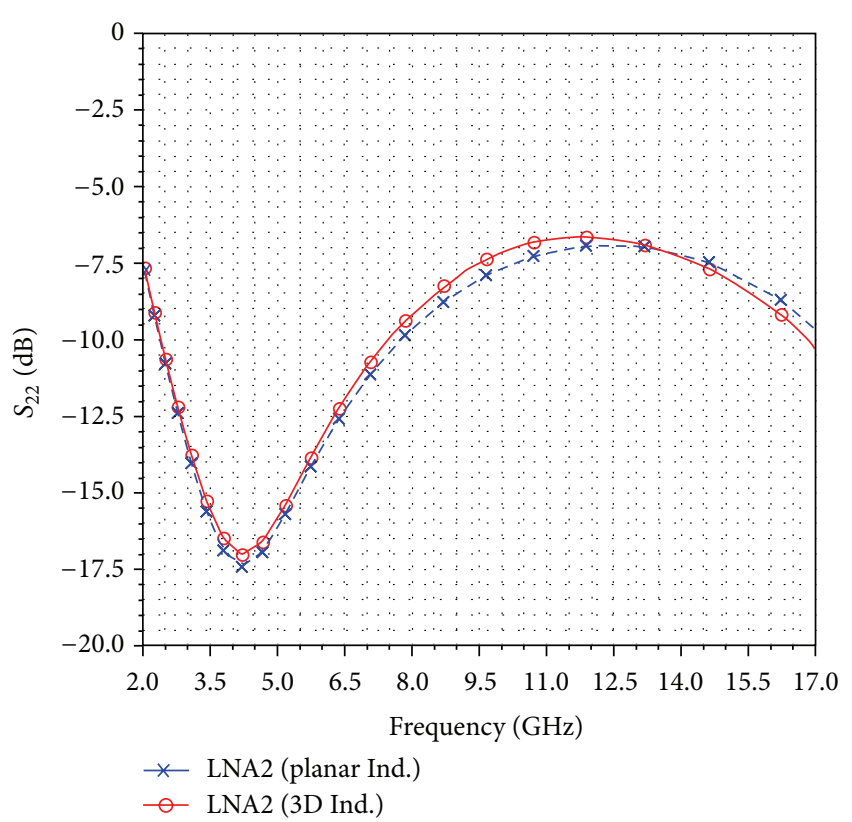

FIGURE 11: $S_{22}(\mathrm{~dB})$ of LNA2.

resonance circuit consisting of capacitor $C_{\text {out }}$ and inductor $L_{\text {out }}$ is used to match the LNA2 output impedance to its load (succeeding RF stage). The resistive termination $R_{\text {out }}$ is used to control the load-output impedance match bandwidth.

A planar RF on-chip spiral inductor $\left(L_{d 1}\right)$ having an inductance of $14.5 \mathrm{nH}$ and a maximum quality factor of 8.0 is needed as a load of the input CS stage to improve the current reuse stages matching. This $\mathrm{RF}$ integrated inductor occupies an area of $428 \mu \mathrm{m} \times 425 \mu \mathrm{m}$ which represents a considerable part of the UWB LNA total die area.

One of the well-known difficulties facing the development of RFICs is inductors large area relative to other passive and active components. This area problem becomes more severe with the recent intensive shrinking of active devices and competitive reduction of fabrication cost [10].

Inductors quality factor $(Q)$ reduction is another limiting factor of RFICs performance enhancement. The reduction of inductor $Q$ factor is due to ohmic and substrate losses. Ohmic losses can be decreased by using a high conductive metal for inductor implementation. On the other hand placing a high resistive layer underneath the inductor can minimize the substrate losses. Lately optimized 3D structures and implementations of RF integrated inductors are suggested to overcome all of these limitations and improve the RF integrated inductors performance $[4,5]$.

For LNA2 circuit area reduction and RF inductor characteristics improvement, a symmetric $3 \mathrm{D}$ structure for $\mathrm{RF}$ integrated inductor implementation is suggested to replace the planar RF integrated inductor $\left(L_{d 1}\right)$. Similar to the design of planar RF inductor, 3D metallic structure layout should be drawn on a substrate to design and test a $3 \mathrm{D}$ integrated inductor [11]. 3D RF inductors structures are mainly consisting of serially connected different metal layers spirals having the same current flow direction. This 3D structure inductance is dependent on these different spirals inductances and the positive mutual coupling they have [11].

For 1P6 M CMOS technology which has six different metal layers, the proposed symmetric $3 \mathrm{D}$ RF integrated inductor has a complete spiral inductor on the highest metal layer (M6) . Half of the lower spiral is implemented using fourth metal layer (M4) to increase its inductance value due to the increased mutual coupling. The second metal layer (M2) which is distant from the top metal layer is employed to implement the lower spiral other half to reduce the parasitic components of that 3D metal structure and increase its quality factor. The suggested symmetric $3 \mathrm{D}$ inductor has an inductance of $14.5 \mathrm{nH}$, a quality factor of 8.5 , and an area of $185 \mu \mathrm{m} \times 165 \mu \mathrm{m}$. $80 \%$ of planar inductor area is saved through this symmetric $3 \mathrm{D}$ structure while achieving the same inductance value and higher quality factor. Figure 5 shows a $3 \mathrm{D}$ view of the proposed symmetric RF integrated inductor.

\section{Simulation Results and Discussion}

The proposed UWB LNA (LNA1 and LNA2) circuits are designed in TSMC CMOS $0.18 \mu \mathrm{m}$ technology process using Agilent Advanced Design System (ADS). Electromagnetic simulation is verified by the post-layout simulation results which are obtained using the Cadence design environment. The suggested symmetric 3D structure is designed and tested using Momentum simulation software and verified using Cadence design environment. The LNAs simulation results are given below.

3.1. Power Gain and Noise Figure. LNA1 has a gain of $17 \pm$ $1.5 \mathrm{~dB}$ as shown in Figure 6. It also has a noise figure less than $2.3 \mathrm{~dB}$ over its operating band of frequency $(3.1-10.6 \mathrm{GHz})$.

$S_{21}(\mathrm{~dB})$ of LNA2 is higher than $10 \mathrm{~dB}$ with a maximum value of $12 \mathrm{~dB}$ over the desired band of frequency (2.5$16 \mathrm{GHz}$ ). This high and flat gain is due to the use of inductive gain-peaking technique in addition to the control of the unity gain current cut-off frequencies of LNA2. Figure 7 shows that the proposed LNA2 employing the symmetric 3D RF integrated inductor achieves a gain of $11 \pm 1.0 \mathrm{~dB}$.

The proposed UWB LNA2 has an enhanced LNA noise performance. LNA2 NF ranges from $2.5 \mathrm{~dB}$ to $3.3 \mathrm{~dB}$ over the operating bandwidth $(2.5-16 \mathrm{GHz})$. This $\mathrm{NF}$ reduction is accomplished due to the optimization of the LNA noise factor given by (4) and the use of weak shunt capacitive-resistive feedback implemented over the input stage. LNA2 achieves a NF less than $3.3 \mathrm{~dB}$ over the operating band of frequency as shown in Figure 8.

3.2. Input and Output Impedance Matching. LNA1 input and output ports have good matching conditions to its source and load, respectively. Simulation results of input and output reflection coefficients of LNA1 are shown in Figure 9. LNA1 has $S_{11}$ and $S_{22}$ less than $-11 \mathrm{~dB}$ and $-10 \mathrm{~dB}$, respectively, over the UWB range of frequencies.

The proposed UWB LNA2 achieves good input impedance matching as shown in Figure 10. Good impedance 
TABLE 1: Proposed UWB LNA performance summery in comparison to recently published UWB LNAs.

\begin{tabular}{lccccc}
\hline Reference & $\begin{array}{c}\text { BW } \\
(\mathrm{GHz})\end{array}$ & $\begin{array}{c}\text { Gain } \\
(\mathrm{dB})\end{array}$ & $\begin{array}{c}\mathrm{NF} \\
(\mathrm{dB})\end{array}$ & $\begin{array}{c}S_{11} \\
(\mathrm{~dB})\end{array}$ & $\begin{array}{c}S_{22} \\
(\mathrm{~dB})\end{array}$ \\
\hline This work (LNA2) $^{*}$ & $\mathbf{2 . 5} \mathbf{1 6}$ & $\mathbf{1 1} \pm \mathbf{1 . 0}$ & $<\mathbf{3 . 3}$ & $<-\mathbf{7}$ & $<-\mathbf{7 . 2 5}$ \\
This work (LNA1) $^{*}$ & $\mathbf{3 . 1} \mathbf{1 0 . 6}$ & $\mathbf{1 7} \pm \mathbf{1 . 5}$ & $<\mathbf{2 . 3}$ & $<-\mathbf{1 1}$ & $<-\mathbf{- 1 0}$ \\
LNA-1 [1] & $1.7 \sim 5.9$ & $11.2 \pm 2.3$ & $<4.7$ & $<-11.8$ & $<-12.7$ \\
LNA-2 [1] & $1.5 \sim 11.7$ & $12.2 \pm 0.6$ & $<4.8$ & $<-8.6$ & $<-10$ \\
[2] & $3 \sim 10.6$ & 15 & $<4.4$ & $<-7$ & NA \\
[12] & $3.1 \sim 10.6$ & $10.8 \pm 1.7$ & $<6$ & $<-10$ & $<-9.3$ \\
[13] & $1 \sim 5$ & $12.7 \pm 0.2$ & $<3.5$ & $<-8$ & NA \\
\hline
\end{tabular}

${ }^{*}$ Post-layout simulation results.

match between LNA2 and its source is obtained using the series-resonant input matching technique. The input return loss $\left(S_{11}\right)$ is less than $-7.0 \mathrm{~dB}$ over this wide range of frequency $(2.5-16 \mathrm{GHz})$.

Figure 11 shows that better output impedance matching is obtained using the planar integrated inductor while simulating LNA2. Good output impedance matching of LNA2 over its operating band of frequency $(2.5-16 \mathrm{GHz})$ is accomplished due to the optimization of the CG output matching stage with the aid of the output LC resonant circuit. $R_{\text {out }}$ termination is used to widen the matching bandwidth. The output return loss $\left(S_{22}\right)$ shown in Figure 11 is less than $-7.25 \mathrm{~dB}$ for LNA2 using the planar inductor while it is less than $-6.0 \mathrm{~dB}$ for LNA2 employing the proposed 3D inductor over the desired frequency band $(2.5-16 \mathrm{GHz})$.

3.3. DC Power, Reverse Isolation, and Stability. LNA1 and LNA2 consume DC power of $12.8 \mathrm{~mW}$ and $20 \mathrm{~mW}$, respectively, from a $1.8 \mathrm{~V}$ power source. The increased DC consumption of LNA2 is due to having enough driving bias for the CG output match stage.

Both of the proposed UWB LNA1 and LNA2 have a reverse isolation factor $\left(S_{12}\right)$ less than $-28 \mathrm{~dB}$ over each design bandwidth. The proposed UWB LNAs (LNA1 and LNA2) are unconditionally stable over their bandwidths.

Table 1 shows a summary of the proposed UWB LNAs performance in comparison to other recently published UWB LNAs implemented in $0.18 \mu \mathrm{m}$ CMOS technology.

\section{Conclusion}

In this paper, two different UWB LNAs were presented. LNA1 has high gain, minimized noise figure, and good impedance match over the UWB range of frequencies. LNA2 has a wide range of operating frequency $(2.5 \mathrm{GHz}-16 \mathrm{GHz})$. UWB LNA2 consists of a current reuse cascaded amplifier with shunt resistive feedback followed by a CG output stage with resistive termination. LNA2 input stage use series-resonant impedance matching technique and employs a symmetric 3D $\mathrm{RF}$ integrated inductor as a load. The post-layout simulation results of LNA1 and LNA2 demonstrate the performance improvement achieved through theses designs. The next step is to implement these UWB LNAs to have a comparison between post-layout simulation results and measured results.

\section{References}

[1] Y. S. Lin, C. Z. Chen, H. Y. Yang et al., "Analysis and design of a CMOS UWB LNA with dual-RLC-branch wideband input matching network," IEEE Transactions on Microwave Theory and Techniques, vol. 58, no. 2, pp. 287-296, 2010.

[2] A. I. A. Galal, R. K. Pokharel, H. Kanay, and K. Yoshida, "Ultrawideband low noise amplifier with shunt resistive feedback in $0.18 \mu \mathrm{m}$ CMOS process," in Proceedings of the 10th Topical Meeting on Silicon Monolithic Integrated Circuits in RF Systems (SiRF '10), pp. 33-36, January 2010.

[3] K. Yousef, H. Jia, R. Pokharel, A. Allam, M. Ragab, and K. Yoshida, "A 2-16 GHz CMOS current reuse cascaded ultrawideband low noise amplifier," in Proceedings of the Saudi International Electronics, Communications and Photonics Conference (SIECPC '11), April 2011.

[4] K. Yousef, H. Jia, R. Pokharel, A. Allam, M. Ragab, and K. Yoshida, "Design of 3D Integrated Inductors for RFICs," in Proceeding of 2012 Japan Egypt Conference on Electronics, Communications and Computers (JECECC '12), pp. 22-25.

[5] X. N. Wang, X. L. Zhao, Y. Zhou, X. H. Dai, and B. C. Cai, "Fabrication and performance of novel RF spiral inductors on silicon," Microelectronics Journal, vol. 36, no. 8, pp. 737-740, 2005.

[6] A. M. Niknejad and R. G. Meyer, "Analysis, design, and optimization of spiral inductors and transformers for Si RF IC's," IEEE Journal of Solid-State Circuits, vol. 33, no. 10, pp. 1470-1481, 1998.

[7] T. H. Lee, The Design of CMOS Radio-Frequency Integrated Circuits, Cambridge University Press, 2nd edition, 2004.

[8] S. S. Mohan, M. Del Mar Hershenson, S. P. Boyd, and T. H. Lee, "Bandwidth extension in CMOS with optimized on-chip inductors," IEEE Journal of Solid-State Circuits, vol. 35, no. 3, pp. 346-355, 2000.

[9] H. T. Friis, "Noise figure of radio receivers," Proceedings of the IRE, vol. 32, no. 7, pp. 419-422, 1944.

[10] H. Y. Tsui and J. Lau, "Experimental results and die area efficient self-shielded on-chip vertical solenoid inductors for multi-GHz CMOS RFIC," in Proceedings of the IEEE Radio Frequency Integrated Circuits (RFIC) Symposium, pp. 243-246, June 2003.

[11] H. Garcia, S. Khemchndai, R. Puildo, A. Lturri, and J. Pino, "A Wideband active feedback LNA with a Modified 3D inductor," Microwave and Optical Technology Letters, vol. 52, pp. 1561-1567, 2010.

[12] P. Sun, Sh. Liao, H. Lin, Ch. Yang, and Y. Hsiao, "Design of 3.1 to 10.6 GHz ultra-wideband low noise amplifier with current reuse techniques and low power consumption," in Proceedings of the Progress In Electromagnetics Research Symposium, pp. 901-905, Beijing, China, March 2009.

[13] A. I. A. Galal, R. K. Pokharel, H. Kanaya, and K. Yoshida, "1-5GHz wideband low noise amplifier using active inductor," in 2010 IEEE International Conference on Ultra-Wideband, ICUWB2010, pp. 193-196, chn, September 2010. 

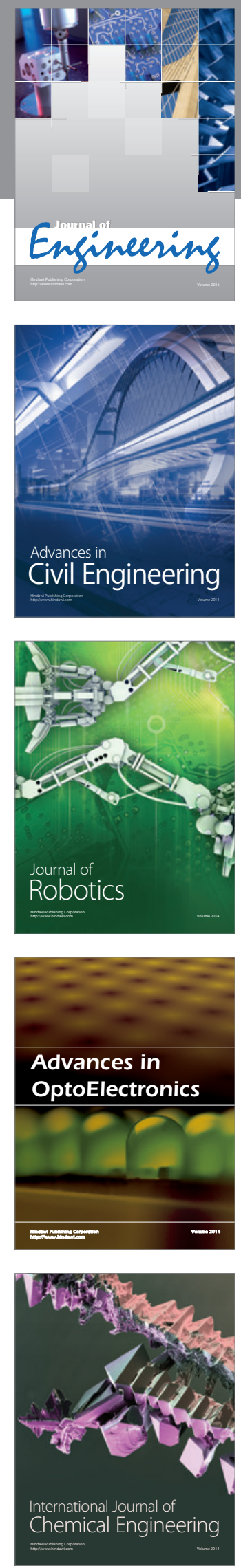

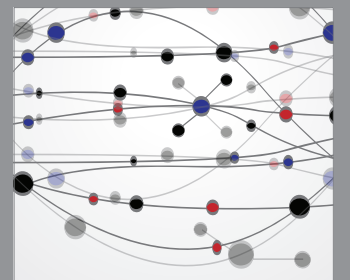

The Scientific World Journal
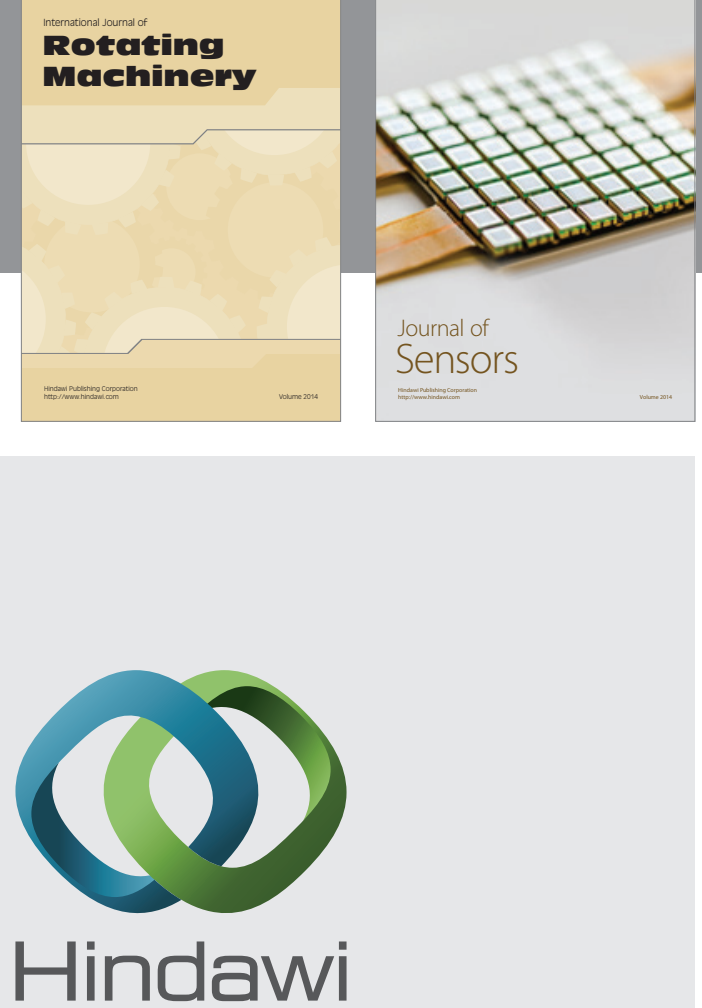

Submit your manuscripts at http://www.hindawi.com
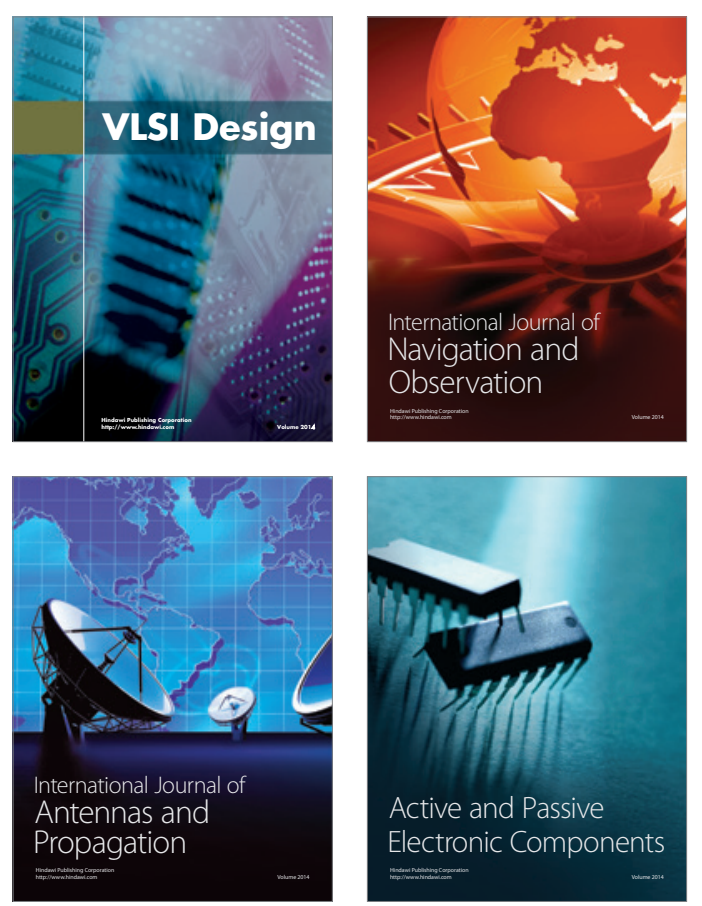
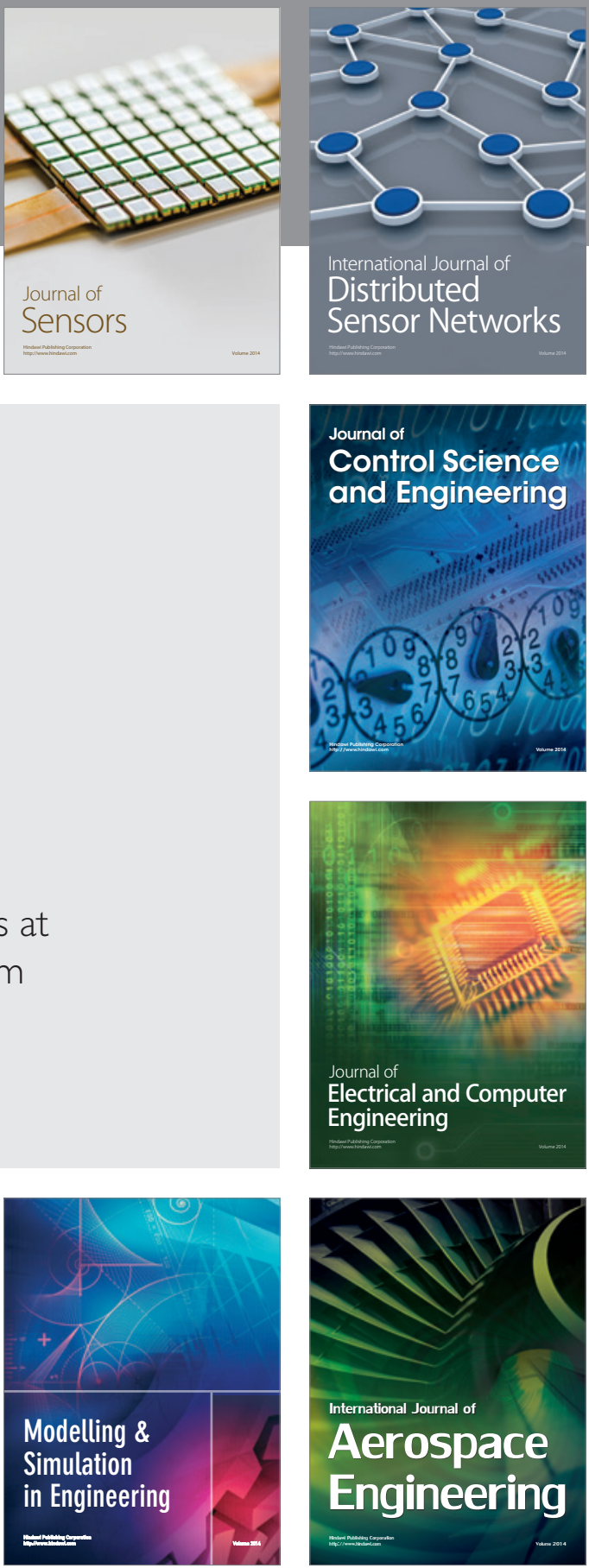

Journal of

Control Science

and Engineering
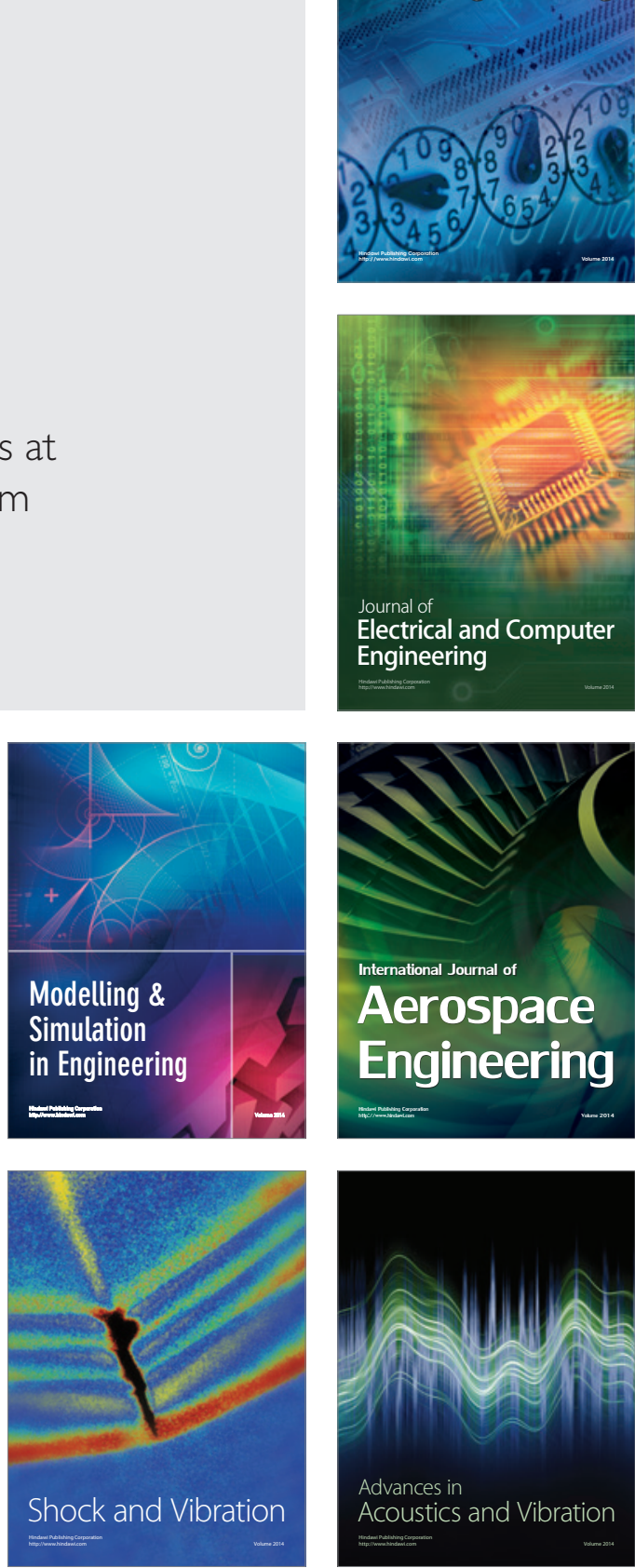